\title{
A Panel of CSF and Serum Biomarkers Reflecting Injury Severity and Outcome in a Human Spinal Cord Injury Study
}

Zhihui Yang ( $\nabla$ zhihuiyang@ufl.edu )

UF: University of Florida

\section{Yueqiang $\mathrm{Fu}$}

UF: University of Florida

\section{Sehajpreet Kaur}

UF: University of Florida

Iktej Singh Jabbal

UF: University of Florida https://orcid.org/0000-0001-8991-7445

Ahmed Moghieb

UF: University of Florida

Ayah Istanbouli

UF: University of Florida

\section{Zoha Irfan}

UF: University of Florida

Helen M. Bramlett

Leonard M Miller School of Medicine: University of Miami School of Medicine

Robert Logan Patterson

UF: University of Florida

Milin Kurup

UF: University of Florida

Lindsey Morrow

UF: University of Florida

Michael Cohn

UF: University of Florida

Samuel J. Ruiz

UF: University of Florida

\section{Zhiqun Zhang}

UF: University of Florida

Jiepei Zhu

UF: University of Florida

Ronald L. Hayes 
Banyan Biomarkers

\section{Ross Bullock}

Leonard M Miller School of Medicine: University of Miami School of Medicine

\section{W. Dalton Dietrich}

Leonard M Miller School of Medicine: University of Miami School of Medicine

\section{Michael Y. Wang}

Leonard M Miller School of Medicine: University of Miami School of Medicine

\section{Kevin K. W. Wang}

UF: University of Florida

\section{Research Article}

Keywords: biomarker, spinal cord injury, injury severity, outcomes, human spinal cord injury

Posted Date: August 19th, 2021

DOl: https://doi.org/10.21203/rs.3.rs-815248/v1

License: (c) (i) This work is licensed under a Creative Commons Attribution 4.0 International License. Read Full License 


\section{Abstract}

Acute spinal cord injury ( $\mathrm{SCl}$ ) results in catastrophic neurological impairment. We aimed to examine the temporal profile and severity correlation of biomarkers, and their relationship with the American Spinal Injury Association Scale (AIS) improvement in human SCI. $15 \mathrm{SCl}$ and 10 non-SCl healthy subjects were classified according to the initial and discharge AIS grade. Serial cerebrospinal fluid (CSF) and serum samples were collected. Spectrin breakdown products (SBDP) 150, SBDP145, glial fibrillary acidic protein (GFAP), and GFAP breakdown product (GBDP) 38/44K were found to be elevated in the acute phase CSF samples in SCl patients on immunoblotting. SBDP150, ubiquitin C-terminal hydrolase-L1 (UCH-L1), GFAP, S100B, neurofilament light chain protein (NF-L), Tau \& interleukin (IL) -6 were elevated in the acute phase CSF and serum samples on ELISA. CSF SBDP150, UCH-L1, GFAP, S100B and Tau were seen to peak on day 1 after injury, while CSF IL-6 and NF-L peaked on day 5. Serum SBDP150, IL-6, S100B, GFAP, UCHL-1 and Tau peaked on day 1 , while serum NF-L peaked on day 5 post-injury. CSF alpha II-spectrin, SBDP150/145, and GBDP 44-38K levels (by immunoblots), CSF SBDP150, S100B, GFAP, UCHL-1 and Tau (ELISA) and serum UCHL-1 and Tau (ELISA) at specific time points showed SCl severity-correlation. CSF SBDP150, GFAP, and Tau and serum UCHL-1 and Tau (ELISA) were seen to have the best correlation with the severity at discharge. Receiver Operating Characteristic Curve analysis showed that CSF and serum biomarkers (SBDP150, IL-6, S100B, GFAP, NF-L, UCHL-1 and Tau) were associated with the severity of SCl.

\section{Introduction}

Traumatic spinal cord injuries (SCl), caused by contact sports, falls, and other accidents, are intense and devastating to many including the patients impacted, as well as their families, friends, and caretakers [1]. While these injuries are relatively infrequent, their impact is strong thus necessitating further research in this field. Initially, there is a primary injury, resulting from mechanical impact or compression from some physical injurious process [2]. While imaging techniques and neurological behavior tests do provide appreciable context to the scope and severity of the injury, it is more helpful to assign concrete values to chemical compounds that present themselves in this context. Our research focuses on the perspective of the secondary injury process, as we evaluate the biochemical cascade that results from the primary injury involving the proteolysis of several cytoskeletal and membrane proteins. There is hope that biofluidbased biomarker tests for neurological injuries would have great potential in the diagnostic process [3].

Several potential biomarkers of central nervous system injury have been identified from cerebrospinal fluid (CSF) and serum in both animal and human studies. The all-spectrin protein forms part of the axolemmal cytoskeleton. The 280-kDa all-spectrin molecule is cleaved into all-spectrin breakdown products (SBDPs) which can be used to detect apoptosis[3,4]. Previous studies have shown significant elevations in the levels of SBDP150 in CSF and serum in the first 24 hours of SCl in rats.[5,6]. Glial fibrillary acidic protein (GFAP) is another biomarker which is a skeletal intermediate filament protein in astrocytes. It reflects the pathological state of astrogliosis and astrocyte injury. Previous studies have shown elevated GFAP levels in CSF after SCI in humans[7,8] and in serum[9] after SCl in rat models, in the acute phase post-injury. S100B, an astroglial $11 \mathrm{kDa}$ calcium-binding protein found in glial cells, can 
reflect the degree of SCl and could be a potential biomarker for the prognosis of acute SCI [10]. Lee et al. demonstrated that raised serum S100B levels in all patients with acute spinal fracture without head injury [11]. Neurofilament light chain (NF-L) present in the axonal cytoplasm, marks neurodegeneration and is often used to evaluate neurological disorders. Its use as a marker in neurotrauma is still an underdeveloped theory. The serum axonal injury marker, phosphorylated neurofilament-H (pNF-H) is another popularly discussed marker for understanding SCI $[12,13]$. Tau protein is a neuronal scaffolding protein, higher levels of which have been correlated with higher injury severities and have been used to predict future neurological outcome in SCI patients [7]. Previously, an animal study has shown that levels in serum and CSF might be promising in predicting the severity and functional outcome of traumatic spinal cord injury [14]. Interleukin (IL)-6, an inflammatory cytokine, has shown associations with injury severity and outcomes following injury [7,15]. Lastly, ubiquitin C-terminal hydrolase-L1 (UCH-L1) is a protein that mainly resides in the neuronal cell body cytoplasm and can reflect neuronal cell body injury. It has been frequently reported as a potential biomarker in traumatic brain injury (TBI) studies, but has not been well studied in SCl. Yang et al. found UCH-L1 levels in both CSF and serum to increase at $4 \mathrm{~h}$ after injury in rat SCl model, followed by an immediate decline [5].

Unfortunately, clinical research in SCl struggles to progress and even pathophysiological mechanisms are unclear [16]. There is therefore an urgent need to accurately assess the severity of injuries, early in their presentation, to predict the functional prognosis, and eventually curate individualized treatment plans for these patients. To accurately diagnose traumatic neural injuries, be it TBI or $\mathrm{SCl}$, it is helpful to understand their pathophysiology. In this study, we explored the dynamic changes in CSF and serum levels of 10 protein biomarkers from days 1 to 5 post-injury by using immunoblots and ELISA, to identify a series of pathologies including neuronal, astroglial and axonal injuries, and neuroinflammation. Identifying these biomarkers will allow us to stratify SCI patients into diagnoses based on the initial severity of neurological impairment and to predict neurological and functional recovery, so that appropriate treatment regimens can be assigned.

\section{Materials And Methods}

\section{Participants and controls}

Consented adult spinal cord injury ( $\mathrm{SCl}$ ) subjects $(\mathrm{N}=15$, mean age was $38.5 \pm 17.1$ years, 13 males) presenting to the Department of Neurological Surgery, Leonard M. Miller School of Medicine of University of Miami, FL were enrolled between September 2011 and August 2015 (IRB\#20090655). Patients were classified according to the American Spinal Injury Association scale (AIS) of impairment on admission (degree of impairment), and, AIS on discharge (degree of improvement). Serial CSF (by lumbar puncture) and serum samples were collected at various time points after injury for biomarker analysis (Univ. Miami Hospital) (day 1-5).

The control CSF and blood serum samples $(n=10)$ were purchased from BiolVT (previous Bioreclamation Inc, Westbury, NY, USA), and were used to compare with archived de-identified SCI CSF and blood serum 
samples $(n=15)$ for the following biomarkers.

\section{Quantitative Immunoblotting}

$10 \mu \mathrm{L}$ control and SCI CSF samples were mixed with SDS sample buffer (50mM Tris, pH 6.8, 25 mM DTT, $2.5 \%$ SDS, $0.02 \%$ bromophenol blue, and $25 \%$ glycerol). Equal amounts of protein were loaded onto Tris/glycine gels (Invitrogen Life Technologies) and then separated by electrophoresis at $200 \mathrm{~V}$ for 60 min. Proteins were transferred to a polyvinylidene difluoride (PVDF) membrane (Invitrogen) using the iBlot Gel Transfer Device (Invitrogen) for $7 \mathrm{~min}$. Following the transfer, the membranes were blocked in 5\% nonfat dry milk in TBST (20 mM Tris- $\mathrm{HCl}, 150 \mathrm{mM} \mathrm{NaCl}$, and 0.003\% Tween-20, pH 7.5) for an hour. Monoclonal anti-mouse all-spectrin (Enzo Life Sciences, NY, USA), anti-spectrin antibody and polyclonal anti-rabbit GFAP (Abcam, MA, USA) were incubated with immunoblotting membranes at a dilution of 1:1000 in $5 \%$ milk at $4{ }^{\circ} \mathrm{C}$ overnight. On the following day, the membranes were washed three times with tris-buffered saline with $0.1 \%$ Tween 20 (TBST) and probed with an alkaline phosphatase-conjugate goat secondary antibody (EMD Millipore, MA, USA) at a dilution of 1:5000 in 5\% milk for an hour, followed by TBST washing. Immunoreactivity was detected using 5-bromo-4-chloro-3- indolyl phosphate (BCIP)/nitro blue tetrazolium phosphatase substrate (Kirkegaard \& Perry Laboratories, Gaithersburg, MD, USA). Band intensity was quantified by NIH ImageJ v1.7 software.

\section{Enzyme-Linked Immunosorbent Assay_(ELISA).}

All assays were conducted according to the manufacturer protocols. The following commercial ELISA kits were used: SBDP150 (cat\# MBS7606326, MyBioSource, Inc, S100B (cat\# EZHS100B-33K, EMD Millipore), and IL-6 (cat\# R6000B, R\&D System). Briefly, standards and 5-10 $\mu \mathrm{L}$ CSF or 30-50 $\mu \mathrm{L}$ serum samples were added into specific antibody pre-coated plates. Then, biotin-conjugated antibodies followed by HRP conjugated avidin were incubated. A signal was detected with a chromogenic substrate (3-3-5-5tetramethylbenzidine (TMB)), and the intensity was measured using a colorimetric plate reader $(450 \mathrm{~nm})$.

\section{Quanterix SIMOA N4PB digital ELISA}

For GFAP, UCH-L1, NF-L and Tau assays, GFAP, UCH-L1, NF-L and Tau concentrations were measured using the same batch of reagents by investigators blinded to clinical data using SIOMA neuro 4 plex kit in SR-X immunoassay analyzer (Quanterix Corp, Boston, MA, USA), which runs ultrasensitive paramagnetic bead-based enzyme-linked immunosorbent assays. Quanterix Simoa N4PB kit measures GFAP, UCH-L1, TTau, and NF-L serum concentrations on a multiplex array simultaneously, according to manufacturer's instructions (https://www.quanterix.com/products-technology/assays/neuro-4-plex-b). Assay methodology was described in detail elsewhere similar to the Quanterix N4PA assay.

$\underline{\text { Statistical Analysis }}$

The data was analyzed using GraphPad Prism and IBM SPSS statistics version 23. The demographic data was presented as median and interquartile range. Mann-Whitney $\mathrm{U}$ and Kruskal-Wallis tests were conducted to assess differences between groups for continuous variables. The accuracy of biomarker 
levels to differentiate between good and poor outcome was evaluated by the receiver operating characteristic (ROC) analysis. All tests were two-tailed, with a significance level set at $5 \%$.

\section{Results}

A total of $15 \mathrm{SCl}$ ( 13 male) patients were included in the study. The mean age was $38.5 \pm 17.1$ years. 8 patients had a cervical $\mathrm{SCl}, 6$ had a thoracic level of $\mathrm{SCl}$, and, 1 had the spinal cord injured at C7-T1 level. On admission, 9 patients on admission had an AIS score of A and 6 had a score B. 4 patients showed an improvement of these scores on discharge. Demographic data and neurologic evaluation (ASIA scores) performed at admission and discharge have been shown in Table 1.

Table 1. Demographic and clinical profile of the cohort of 15 patients with Spinal cord injury 


\begin{tabular}{|c|c|c|c|c|c|c|c|}
\hline $\begin{array}{l}\text { Patient } \\
\#\end{array}$ & Age & Gender & Injury mode & $\begin{array}{l}\text { Injury } \\
\text { site }\end{array}$ & $\begin{array}{l}\text { Initial } \\
\text { AIS }\end{array}$ & $\begin{array}{l}\text { Discharge } \\
\text { AIS }\end{array}$ & $\begin{array}{l}\text { AIS } \\
\text { improvement } \\
\text { by grade }\end{array}$ \\
\hline P1 & 43 & $M$ & $\begin{array}{l}\text { Pedestrian motor } \\
\text { vehicle accident }\end{array}$ & $\begin{array}{l}\text { C7- } \\
\text { T1 }\end{array}$ & A & $A$ & 0 \\
\hline P2 & 38 & $M$ & Fall & $\begin{array}{l}\text { T3- } \\
\text { T4 }\end{array}$ & B & $\mathrm{D}$ & 2 \\
\hline P3 & 21 & $M$ & Diving accident & $\begin{array}{l}\text { C5- } \\
\text { C6 }\end{array}$ & B & $\mathrm{D}$ & 2 \\
\hline P4 & 28 & $M$ & Diving accident & $\begin{array}{l}\text { C5- } \\
\text { C6 }\end{array}$ & B & $\mathrm{B}$ & 0 \\
\hline P5 & 19 & $M$ & $\begin{array}{l}\text { motor vehicle } \\
\text { accident }\end{array}$ & $\begin{array}{l}\text { C5- } \\
\text { C6 }\end{array}$ & B & $\mathrm{C}$ & 1 \\
\hline P6 & 22 & $M$ & Sport Injury & C5 & A & $A$ & 0 \\
\hline P7 & 48 & $M$ & Motorcycle accident & T3 & A & $A$ & 0 \\
\hline P8 & 38 & $\mathrm{~F}$ & $\begin{array}{l}\text { Motor vehicle } \\
\text { accident }\end{array}$ & $\mathrm{T} 1 / 2$ & A & $A$ & 0 \\
\hline P9 & 29 & $\mathrm{~F}$ & $\begin{array}{l}\text { Motor vehicle } \\
\text { accident }\end{array}$ & T12 & A & $A$ & 0 \\
\hline P10 & 57 & $M$ & Fall & $\begin{array}{l}\text { T11- } \\
12\end{array}$ & A & $A$ & 0 \\
\hline P11 & 67 & $M$ & Fall & $\begin{array}{l}\text { C3- } \\
\text { C4 }\end{array}$ & A & A (Dead) & 0 \\
\hline P12 & 22 & $M$ & Sport Injury & $\begin{array}{l}\text { C5- } \\
\text { C6 }\end{array}$ & A & $B$ & 1 \\
\hline P13 & 66 & $M$ & Fall & $\begin{array}{l}\text { T12- } \\
\text { L1 }\end{array}$ & B & $B$ & 0 \\
\hline P14 & 22 & $M$ & Gunshot & $\mathrm{T}-12$ & A & $A$ & 0 \\
\hline P15 & 58 & $M$ & Fall & $\begin{array}{l}\text { T4- } \\
\text { T5 }\end{array}$ & B & $B$ & 0 \\
\hline
\end{tabular}

CSF Biomarkers in human SCI by Immunoblotting

Immunoblotting detected CSF levels of neuronal all-spectrin $(280 \mathrm{kDa})$, its breakdown products SBDP150 and SBDP145, astroglial GFAP and its breakdown products GBDP-44 and GBDP-38. Immunoblots for serial CSF samples from three representative subjects are shown in Fig1a. Compared to the normal control, SBDP150/145 levels rising to a peak on day 1 followed by a decline from day 2 to 5 (Fig1 b-c). While intact GFAP was barely detectable, GBDP-38K and $-44 \mathrm{~K}$ appeared prominently in SCI samples. 
GBDP-38K and -44K levels were the highest on day 1, declined on days 2 and 3 , and returned to the control levels on days 4 and 5 (Fig1d-e).

We also attempted to correlate SCl severity with these CSF biomarker levels. Patients with an initial AIS score A were seen to have a significantly higher level of CSF all-spectrin on day 4 as compared to those with an initial AIS score B (Fig2). CSF SBDP150/145 levels of patients with an initial AIS score A on day 2 and 3 was significantly higher than those with an initial AIS B. CSF GBDP-44 -38K on day 1, 2 and 3 were higher in patients with initial AIS score A versus patients with initial AIS score B significantly, respectively (Fig2). Similarly, CSF all-spectrin SBDP150/145 levels on days 3 and 4 in patients with AIS A on discharge were higher than those in patients with AIS scores B-D. In patients having an AIS score A on discharge, the levels of CSF GFAP on day 5 and of its breakdown products (CSF GBDP-44-38K) on day 3, 4 and 5 were higher than those with AIS scores of B-D on discharge (Online Resource 1). Lastly, we also examined the correlation of CSF biomarker levels with AIS improvement by grade ( $\triangle$ AIS $=$ discharge AIS initial AIS ). There was a trend for higher biomarker levels in the no-improvement group $(\triangle A I S=0)$ when compared with those with 1 or 2 grade improvement $(\triangle A I S=1-2)$ for SBDP150, GFAP and GBDP 38-44K as on day 5 . But the difference was not statistically significant (Fig3).

\section{CSF Biomarkers in human SCI by ELISA}

To achieve more quantitative data, we also assayed human control and SCI CSF samples for additional biomarkers by ELISA, including SBDP150, UCH-L1, GFAP, S100B, NF-L, Tau and IL-6. We observed that the levels of all CSF markers were elevated in the acute phase. SBDP150 levels peaked on day 1 and decreased after that. UCH-L1 showed the highest initial increase on day 1 compared to control levels and then gradually decreased to show a slight increase again on day 5. GFAP and S100B levels were the highest on day 1 post-injury, remaining elevated up to day 5. Tau peaked 1 day after injury, stabilized on the subsequent day and then declined to control levels. NF-L significantly increased on day 1 and maintained its levels before peaking on day 5. Similar to NF-L, IL-6 increased on day 1 and peaked on day 5 , though not as much as NF-L did (Fig4). All biomarkers were significantly elevated on day 1 when compared to healthy controls. Receiver operating characteristic (ROC) curves were used to assess the diagnostic values of biomarker levels based on area under the curve (AUC, ranging from 0.784 to 0.977 ) for distinguishing healthy controls from day $1 \mathrm{SCl}$ levels as showed in Online Resource 2. CSF ROC curves are shown in Online Resource 3. CSF NF-L levels had the best AUC (0.9769) among all six markers examined.

We also examined if CSF biomarkers were sensitive to initial AIS score A versus B (Fig 5). Compared to SCI patients with initial AIS B, SBDP150 on days 3 and 4 were significantly higher in those with initial AIS A. Similarly, S100B and GFAP on days 2 and 3 were higher in patients with initial AIS A versus patients with initial AIS B, respectively. UCHL-1 of patients with initial AIS A on day 2 was significantly higher than those with initial AIS B. NF-L on day 4 to 5 and Tau on days 2, 3 and 5 in patients with initial AIS A were found to be higher than those in patients with initial AIS B. Similarly, we also examined if CSF biomarkers were sensitive to discharge AIS score A vs. B-D (Online Resource 4). SBDP150 levels on day 4 of SCI 
patients with discharge AIS A were significantly higher than those with discharge AIS B to D. GFAP and Tau on day 3 were higher in patients with discharge AIS A versus those with discharge AIS B-D. Compared to patients with discharge AIS B-D, NF-L at day 4 was higher in patients with discharge AIS A, although not statistically significant. Another way to examine if CSF biomarkers could prognosticate SCI patient improvement at discharge, we compared biomarker levels in patients with no improvement from admission to discharge ( $\triangle$ AIS 0 ) to those with at least one grade improvement (e.g. from $A$ to $B$ or from $B$ to D) ( $\triangle$ AIS 1-2). However, we found no statistically significant differences between the CSF levels of any biomarker on any day between patients with and without AIS improvement (Fig6).

\section{Serum Biomarkers in Human SCI by ELISA}

We also examined biomarker levels in serial serum samples from the $\mathrm{SCl}$ patients versus healthy controls. By ELISA, serum levels of SBDP150, UCH-L1, GFAP, S100B, NF-L, Tau and IL-6 were found to be elevated in the acute phase serum samples from SCI patients (Fig7). Serum SBDP150, IL-6 and GFAP levels were highest on day 1 and were still significantly elevated from day 2 to day 5 . Serum UCHL-1 levels peaked on day 1 and then decreased from day 2 to 5 . Serum Tau was significantly elevated on days 1 and 2 and declined from days 3 to 5 . Serum NF-L levels were elevated in the acute phase, but reached the maximum on day 5. Serum S100B levels were highest on day 1, decreased from day 2 to 4 , and returned to control levels on day 5. Receiver operating characteristic (ROC) curves were used to assess the diagnostic values of serum biomarkers on day 1 in patients with $\mathrm{SCl}$ (Online Resource 5). Area under the curve, $A \cup C$, ranged from 0.81 to 1.00 , was used in distinguishing healthy controls from $\mathrm{SCl}$ day 1 samples (Online Resource 6). Serum NF-L, SBDP150 and IL-6 had the best AUC (1.00). Thus, all serum biomarkers examined are able to diagnose $\mathrm{SCl}$ in ROC statistics.

We also examined if serum biomarkers were sensitive to initial AIS score A versus B (Fig8). Compared to SCl patients with initial AIS B, serum GFAP, UCHL- 1 and Tau on days 2, 4 and 5 were higher in patients with initial AIS A. Serum SBDP150 on days 2 and 4 in patients with initial AIS A were higher than those in with initial AIS B. However, there were no statistically significant differences between SCI patients with initial AIS A versus those with initial AIS B in serum S100B, IL-6 and NF-L on any day. Similarly, we also examined if serum biomarkers were sensitive to discharge AIS score A vs. B (Online Resource 7). Serum GFAP, UCHL- 1 and Tau levels on days 2 and 4 for patients with discharge AIS A were higher than those of $\mathrm{SCl}$ patients with discharge AIS B to D, respectively. However these were statistically significant only for Tau. Compared to patients with discharge AIS B to D, serum SBDP150 on day 3 and S100B on day 2 were higher in patients with discharge AIS A. Moreover, serum Tau levels had the best correlation to the discharge AIS. Lastly, we examined if serum biomarkers could prognosticate improvement of SCI patients based on score improvement from initial to discharge AIS. Compared to patients without AIS improvement at discharge, serum UCHL-1 and Tau on days 2 and 4 were lower in patients with AIS improvement (by 1 or more points in AIS score), at discharge (Fig9).

\section{Discussion}


In this study, we examined a panel of cerebrospinal fluid (CSF) and serum protein biomarkers for their ability to diagnose human spinal cord injury ( $\mathrm{SCl}$ ) severity and to prognosticate discharge outcomes in these patients.

CSF SBDP150 is a biomarker that reflects axonal injury as well as necrosis and apoptosis of brain cells [3]. For our analysis, in addition to quantifying the levels, we compared the trends of CSF alpha-II spectrin and SBDP150 with the 'initial' and 'discharge' AIS grades of patients as well. We found acute SCI to correlate with high CSF SBDP150 levels on immunoblotting and ELISA. This was in accordance with previous studies albeit in rat models. Yang et al. in addition to coming to a similar conclusion on ELISA in rat SCl model, showed a correlation of higher levels of both all-spectrin and SBDPs with increasing severity of injury[5]. In another study, Yokobori et al. also found the levels of both of these biomarkers to be increased in the CSF as early as 4 hours after $\mathrm{SCl}$ in rats [6]. A similar study was performed by Mondello et al in human subjects with TBI, which found significantly higher CSF levels of SBDP 145 in those patients who died than in those who survived the injury[4]. In our knowledge, our analysis is the first attempt to assay SBDP150 in the CSF in humans with SCI.

CSF GFAP which is the most studied biomarker in SCl, reflects injury to astrocytes. On immunoblotting, we found that its breakdown products ( $44 \mathrm{~K}$ to $38 \mathrm{~K}$ ) in the CSF were in fact the dominant species over the intact GFAP after $\mathrm{SCl}$, mirroring a previous pilot study by Yokobori et al [6]. Additionally, CSF GFAP levels were significantly higher in patients with a more severe $\mathrm{SCl}$ at presentation (AIS A) on days 2, 3 and 5 (Fig5). Previously, Kwon et al. reported that CSF GFAP detected by ELISA at $24 \mathrm{~h}$ postinjury could predict, with $89 \%$ accuracy, future AIS graded injury severities as well as 6-month postinjury segmental motor improvements in $\mathrm{SCl}$ patients [17]. Moreover Kwon et al. also showed that its levels additionally served as a predictor of future neurological outcomes in 50 acute SCI patients [7]. In another study, CSF GFAP levels were measured within $24 \mathrm{~h}$ after injury in $16 \mathrm{SCl}$ patients and were found to be significantly elevated in $\mathrm{SCl}$ patients when compared to healthy controls [8]. Winnerkvist et al. measured CSF GFAP concentration in 39 patients undergoing elective thoracoabdominal aortic aneurysm surgery and found that patients with paraplegia had significant increases of CSF GFAP, NF-L, and S100B compared with asymptomatic patients during the postoperative period [18]. Ahadi et al found the mean serum levels of GFAP in SCl patients, at 24, 48, and 72-h time-points postinjury, to be significantly higher than healthy control levels [9]. Furthermore, serum GFAP levels in their analysis correlated with injury severity as well. Guez et al., on the contrary, did not find a significant elevation in its CSF concentration in SCl patients when compared to healthy controls [19]. Similar to GFAP, S100B is another biomarker of astroglial injury. Our study found both CSF and serum S100B levels to peak on day 1 . Also, there was a statistically significant increase of levels on days 2, 3 and 4 in patients with initial AIS A as compared to those with initial AIS B. However, we found no significant difference in CSF S100B between patients with discharge AIS grade A and discharge AIS grades B-D. In a previous study, Du et al. reported serum levels of S100B in patients with less than 1, 1 or more than 1 AIS grade improvement at 6 months after the injury to be elevated, reaching a peak on the 4th day, and then declining gradually.[10] Other studies have found S100B higher levels to be correlated with higher injury severities [7,8]. Additionally, Kwon et al. found that its levels predicted future 
neurological outcome in SCI patients as well [7]. Therefore, not only do both of these astroglial markers show a rise over the acute phase of $\mathrm{SCl}$, they can also be used to prognosticate the severity of the injury.

Post-injury, neuronal and astroglial protein biomarkers are likely released through disrupted membranes of the cells of the spinal cord into the interstitial fluid, which then eventually reach the CSF and blood. CSF being closer to the injured spinal cord, reflects the pathological changes more accurately although it is not routinely collected in the clinical management of $\mathrm{SCl}$ patients (unlike in severe traumatic brain injury). Thus, it is more clinically feasible to obtain blood samples through a vascular puncture as compared to obtaining CSF samples through a lumbar puncture in patients. Our study showed that the levels of S100B, GFAP, UCH-L1, NF-L, and Tau analyzed using ELISA were much higher in CSF than those in serum, except for SBDP150 and IL-6. Additionally, the trends of SBDP150, S100B, GFAP, UCH-L1, NF-L and Tau elevations in CSF after injury mirrored those in serum. Additionally, we found all CSF and serum biomarkers on day- 1 to be able to diagnose $\mathrm{SCl}$ by ROC analysis (Online Resources 2 and 6). Moreover, the ability of serum biomarkers to evaluate the initial injury severity and prognosis of SCl patients was similar to that of the corresponding CSF biomarkers. This indicated the possibility of using most of serum biomarkers in this study in the clinical diagnosis and treatment of $\mathrm{SCl}$ patients.

The biomarkers NF-L and Tau reflect axonal injury. NF-L levels in both CSF and serum increased and peaked on day-

5 in SCl patients. While Tau levels in CSF fell back to the baseline by day 5 , levels of NF-L still maintained significantly high levels on the day of discharge. No statistically significant difference of NF-L values was found between patients classified based on injury severity, that is, either according to initial or discharge AIS grades. These findings are in contrast to a previous study by Pouw et al. which indicated that the mean CSF NF-L levels were significantly higher in AIS A and B patients than in AIS C and D patients [8]. Similarly, Guez et al researched that SCI patients with complete as compared to incomplete motor loss showed higher levels of CSF NF-L [19]. Kuhle et al. also reported that elevated serum NF-L levels were associated with a more severe $\mathrm{SCl}$ and poorer neurological prognosis [20]. We believe that the inability of our analysis to identify a correlation of NF-L levels with injury severity can be explained by the fact that $\mathrm{NF}-\mathrm{L}$ is a biomarker of chronic $\mathrm{SCl}$, the levels of which normally take 2-3 weeks to rise significantly after injury [19]. As far as CSF Tau was concerned, in our study, its levels were significantly higher on days 2 and 5 in patients with initial AIS A as compared to those with initial AIS B. A similar pattern was observed with the discharge AIS grades, i.e., A versus B-D. These findings were similar to a previous study in which higher levels of CSF-Tau correlated with higher injury severity [7]. A study by Pouw et al. on the other hand reported no significant correlations between CSF Tau levels and AIS grades in SCI patients [8].

We found the levels of CSF IL-6 did not correlate with the 'initial' or 'discharge' injury severity. This may be because longer follow-up periods are required to study elevations in markers of neuroinflammation like IL6. Yang et al. indicated that SCI injury did not affect IL-6 levels in either CSF or serum in rat models [5]. In contrast, to the Dalkilic et al. reported CSF $1 \mathrm{~L}-6$ at $24 \mathrm{~h}$ post-injury to correlate with injury severities and to 
predict prognosis in SCI patients [15]. Similarly, Kwon et al. found the levels of CSF IL-6 at $24 \mathrm{~h}$ amongst AIS A patients post-injury to be significantly different in those who did and did not improve over time [7].

UCH-L1 biomarker is a protein which reflects neuronal cell body injury. We found both CSF and serum UCH-L1 levels to be highest on day 1 . While serum UCH-L1 significantly correlated with discharge AIS grades (on day 1), CSF UCH-L1 did not. Yokobori et al. reported that CSF and serum UCH-L1 increased when compared to the healthy controls, and both peaked on day-2 post-injury and then declined quickly in $\mathrm{SCl}$ patients [6]. Yang et al. found that UCH-L1 levels in both CSF and serum increased at $4 \mathrm{~h}$ after injury in rat $\mathrm{SCl}$ model, followed by an immediate decline [5]. Furthermore, CSF UCH-L1 levels correlated with the severity of $\mathrm{SCl}[5]$.

Notably, in our study, there was no CSF biomarker that showed a statistically significant difference between SCl patients with an improved AIS score at discharge and those with no improvement (Fig6). However, among the serum biomarkers, only Tau protein levels, on day 2, were significantly higher in SCI patients with no improvement in AIS grade on discharge as compared to those who showed improvement (Fig9).

Our study was not without limitations. Firstly, the study population was relatively small and limited to a single medical center. Further validation in a larger cohort from multiple centers is warranted. Secondly, all time points of sample collection were in the acute phase, while the chronic biomarkers for prognosis of $\mathrm{SCl}$ were not explored. Thirdly, SCl patients in the study were only scored using AIS grade at admission and discharge, and there was no long-term follow-up after discharge. Fourthly, in assessing outcome, there were two groups divided based on discharge AIS grades. However, only one SCI patient with initial AIS A converted to AIS B on discharge. So, there was a large overlap between the initial and the discharge AIS rating groups. Finally, we used different detection methods, such as immunoblotting, ELISA and digital SIMOA N4PB ELISA, to measure the levels of biofluid biomarkers. This may have led to a deviation of results as the sensitivity of each detection method may be different.

Despite these limitations, this is the first report of a systematic exploration of acute biofluid biomarkers involving neuronal, astroglial, axonal injuries, and neuroinflammation in a clinical human $\mathrm{SCl}$ study. Our study is the first comprehensive examination of biofluid biomarkers that are expressed in the CSF and serum during the acute phase of traumatic $\mathrm{SCl}$ in humans. Taken together, our results suggest that when used together, these CSF and serum biomarkers not only help reveal the potential mechanisms of $\mathrm{SCl}$, but also evaluate injury severity at initial presentation and discharge and help predict disease progression.

\section{Declarations}

\section{Funding}

This work was supported by grant W81XWH-12-1-0276 and W81XWH-12-1-0277 from the United States Army (Department of Defence). 


\section{Conflicts of Interest/Competing interests}

Author Disclosures: Author RLH is s shareholder and employee of Banyan Biomarker. Author KKW is a shareholder of Gryphon Bio Inc. and Banyan Biomarkers Inc.

\section{Availability of data and material}

The data that support the findings of this study are available upon reasonable request from the corresponding author.

\section{Code availability}

Not applicable.

\section{Author Contributions}

All authors contributed to the design and implementation of the research, to the analysis of the results and to the writing of the manuscript. All authors read and approved the final manuscript.

\section{Ethics approval}

The study was approved by the institutional ethics committee of the Leonard M. Miller School of Medicine of University of Miami, FL (IRB\#20090655). The authors certify that the study was performed in accordance with the ethical standards as laid down in the 1964 Declaration of Helsinki and its later amendments.

\section{Consent to participate}

Informed consent was obtained from all individual participants included in the study.

\section{Consent for publication}

The authors affirm that human research participants provided informed consent for publication of their data.

\section{Acknowledgements}

We thank Amy Paulino and Michael Catania (Banyan Biomarkers) for logistic and technical biomarker support.

\section{References}

[1] C. S. Ahuja et al., "Traumatic spinal cord injury," Nature Reviews Disease Primers, vol. 3. Nature Publishing Group, Apr. 27, 2017. doi: 10.1038/nrdp.2017.18. 
[2] M. J. Eckert and M. J. Martin, "Trauma: Spinal Cord Injury," Surgical Clinics of North America, vol. 97, no. 5. W.B. Saunders, pp. 1031-1045, Oct. 01, 2017. doi: 10.1016/j.suc.2017.06.008.

[3] K. K. Wang et al., "An update on diagnostic and prognostic biomarkers for traumatic brain injury," Expert Review of Molecular Diagnostics, vol. 18, no. 2. Taylor and Francis Ltd, pp. 165-180, Feb. 01, 2018. doi: 10.1080/14737159.2018.1428089.

[4] S. Mondello et al., "ail-spectrin breakdown products (SBDPs): Diagnosis and outcome in severe traumatic brain injury patients," Journal of Neurotrauma, vol. 27, no. 7, pp. 1203-1213, Jul. 2010, doi: 10.1089/neu.2010.1278.

[5] Z. Yang et al., "Temporal Profile and Severity Correlation of a Panel of Rat Spinal Cord Injury Protein Biomarkers," Molecular Neurobiology, vol. 55, no. 3, pp. 2174-2184, Mar. 2018, doi: 10.1007/s12035-017-0424-7.

[6] S. Yokobori et al., "Acute diagnostic biomarkers for spinal cord injury: Review of the literature and preliminary research report," World Neurosurgery, vol. 83, no. 5. Elsevier Inc., pp. 867-878, May 01, 2015. doi: 10.1016/j.wneu.2013.03.012.

[7] B. K. Kwon et al., "Cerebrospinal fluid biomarkers to stratify injury severity and predict outcome in human traumatic spinal cord injury," Journal of Neurotrauma, vol. 34, no. 3, pp. 567-580, Feb. 2017, doi: 10.1089/neu.2016.4435.

[8] M. H. Pouw et al., "Structural biomarkers in the cerebrospinal fluid within $24 \mathrm{~h}$ after a traumatic spinal cord injury: A descriptive analysis of 16 subjects," Spinal Cord, vol. 52, no. 6, pp. 428-433, 2014, doi: 10.1038/sc.2014.26.

[9] R. Ahadi, F. Khodagholi, A. Daneshi, A. Vafaei, A. A. Mafi, and M. Jorjani, “Diagnostic Value of Serum Levels of GFAP, pNF-H, and NSE Compared with Clinical Findings in Severity Assessment of Human Traumatic Spinal Cord Injury," Spine, vol. 40, no. 14, pp. E823-E830, Jul. 2015, doi: 10.1097/BRS.0000000000000654.

[10] W. Du et al., "The prognostic value of serum neuron specific enolase (NSE) and s100b level in patients of acute spinal cord injury," Medical Science Monitor, vol. 24, pp. 4510-4515, Jun. 2018, doi: 10.12659/MSM.907406.

[11] S. J. Lee et al., "Elevated serum S100B levels in acute spinal fracture without head injury," Emergency Medicine Journal, vol. 27, no. 3, pp. 209-212, Mar. 2010, doi: 10.1136/emj.2008.063743.

[12] G. Shaw et al., "Hyperphosphorylated neurofilament NF-H is a serum biomarker of axonal injury," Biochemical and Biophysical Research Communications, vol. 336, no. 4, pp. 1268-1277, Nov. 2005, doi: 10.1016/j.bbrc.2005.08.252. 
[13] N. J. Olby et al., "Time course and prognostic value of serum GFAP, pNFH, and S100 $\beta$ concentrations in dogs with complete spinal cord injury because of intervertebral disc extrusion," Journal of Veterinary Internal Medicine, vol. 33, no. 2, pp. 726-734, Mar. 2019, doi: 10.1111/jvim.15439.

[14] Y. Tang et al., "Serum and cerebrospinal fluid tau protein level as biomarkers for evaluating acute spinal cord injury severity and motor function outcome," Neural Regeneration Research, vol. 14, no. 5, pp. 896-902, May 2019, doi: 10.4103/1673-5374.249238.

[15] T. Dalkilic et al., "Predicting Injury Severity and Neurological Recovery after Acute Cervical Spinal Cord Injury: A Comparison of Cerebrospinal Fluid and Magnetic Resonance Imaging Biomarkers," Journal of Neurotrauma, vol. 35, no. 3, pp. 435-445, Feb. 2018, doi: 10.1089/neu.2017.5357.

[16] B. K. Kwon et al., "Neurochemical biomarkers in spinal cord injury," Spinal Cord, vol. 57, no. 10. Nature Publishing Group, pp. 819-831, Oct. 01, 2019. doi: 10.1038/s41393-019-0319-8.

[17] B. K. Kwon et al., "Cerebrospinal fluid inflammatory cytokines and biomarkers of injury severity in acute human spinal cord injury," Journal of Neurotrauma, vol. 27, no. 4, pp. 669-682, Apr. 2010, doi: 10.1089/neu.2009.1080.

[18] A. Winnerkvist et al., "Multilevel somatosensory evoked potentials and cerebrospinal proteins: indicators of spinal cord injury in thoracoabdominal aortic aneurysm surgery," European Journal of Cardio-thoracic Surgery, vol. 31, no. 4, pp. 637-642, Apr. 2007, doi: 10.1016/j.ejcts.2007.01.007.

[19] M. Guéz, C. Hildingsson, L. Rosengren, K. Karlsson, and G. Toolanen, "Nervous tissue damage markers in cerebrospinal fluid after cervical spine injuries and whiplash trauma," Journal of Neurotrauma, vol. 20 , no. 9 , pp. $853-858$, Sep. 2003 , doi: $10.1089 / 089771503322385782$.

[20] J. Kuhle et al., "Serum neurofilament light chain is a biomarker of human spinal cord injury severity and outcome," Journal of Neurology, Neurosurgery and Psychiatry, vol. 86, no. 3, pp. 273-279, Mar. 2015, doi: 10.1136/jnnp-2013-307454.

\section{Figures}


a
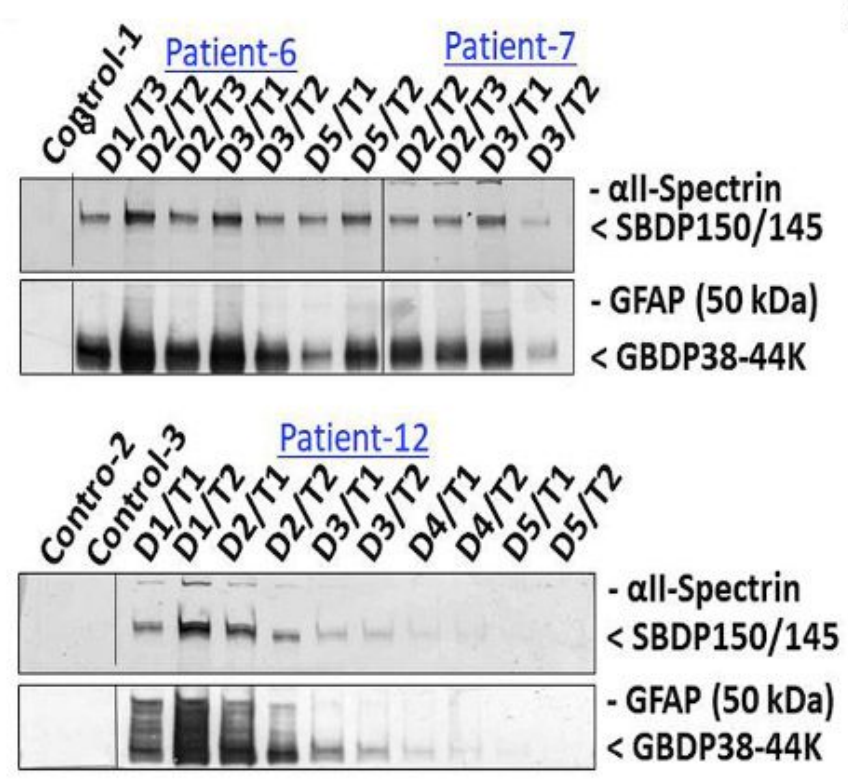

b

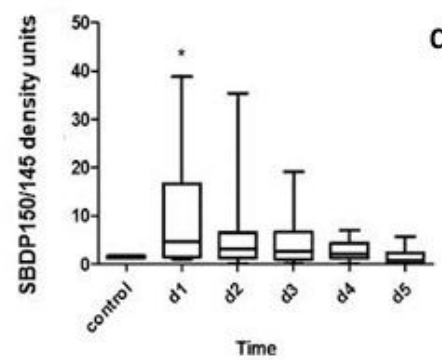

C

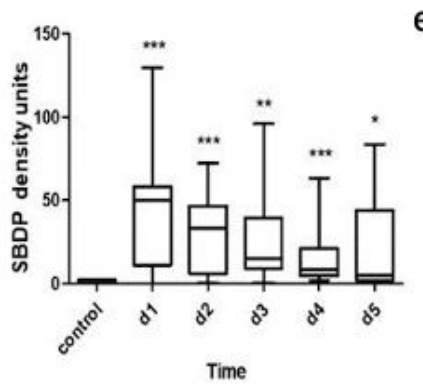

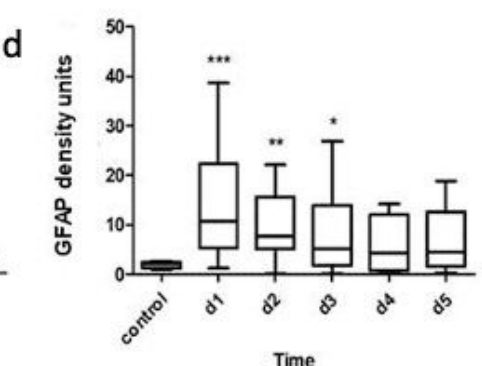

e

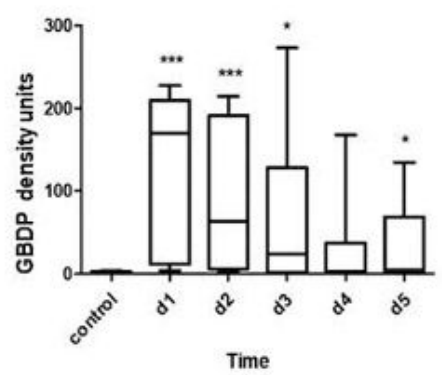

Figure 1

Neuronal alpha II spectrin, its breakdown products (SBDP), GFAP and its breakdown products (GBDP) levels in human cerebrospinal fluid (CSF) after spinal cord injury (SCI), by Immunoblotting a:

Representative images from immunoblotting for all-Spectrin, SBDP, GFAP and GBDP; $b$ to e: Quantification shown as median, interquartile range (box) and upper and lower values (whiskers) for alpha II spectrin (B), SBDP150/145 K (C), GFAP (D) and GFAP-44K-38K (E). * **, *** $p$ values $<0.05,0.01$, 0.001 , respectively vs. control. 

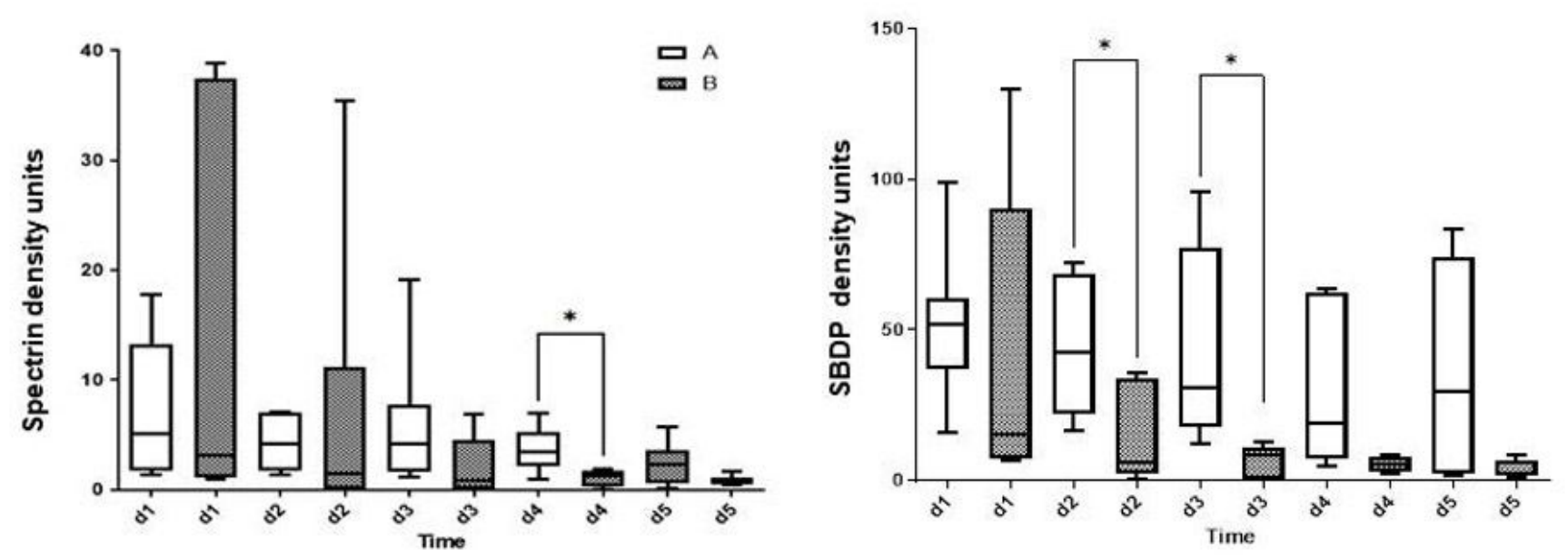

$\square A$
$B$
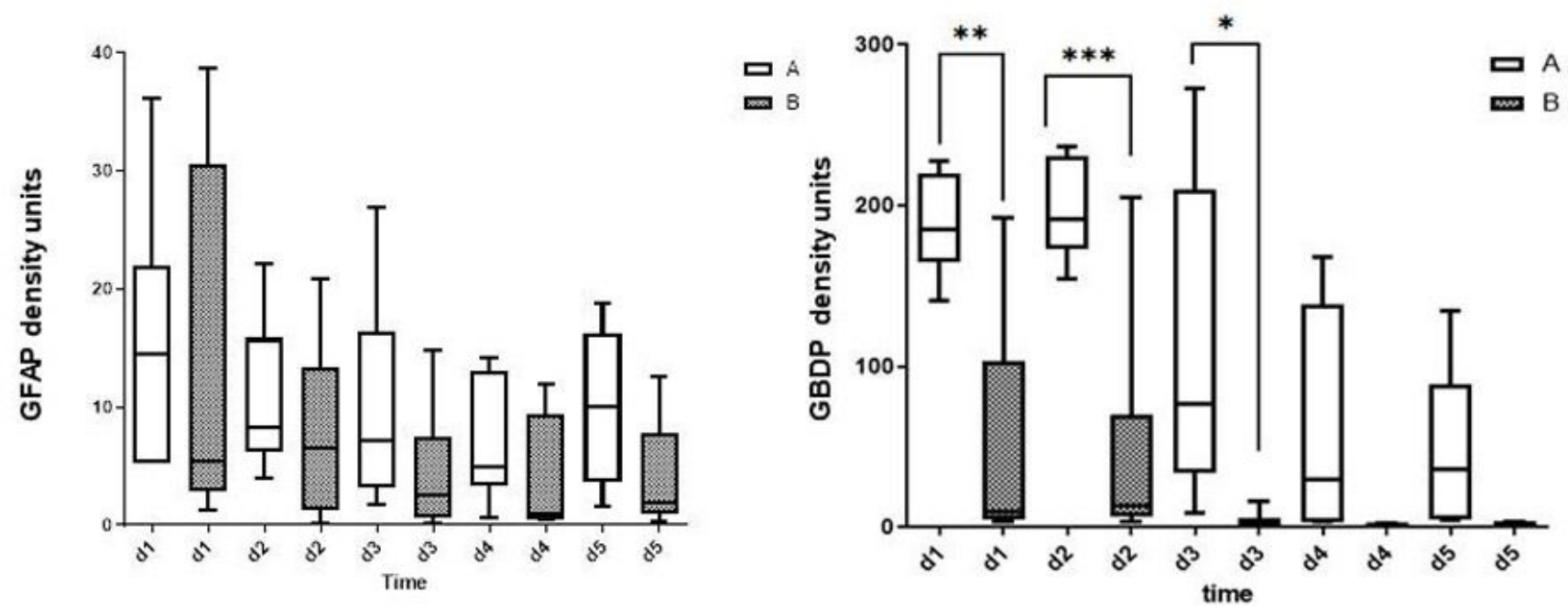

Figure 2

Correlation of CSF biomarker values to injury severity (initial AIS) Biomarkers measured with Immunoblotting. Quantification shown as mean + SEM for alpha II-spectrin, SBDP150/145, GFAP and GFAP-44K-38K. * $* \star * * \star * ~ p$ values $<0.05,0.01,0.001$, respectively between groups. 

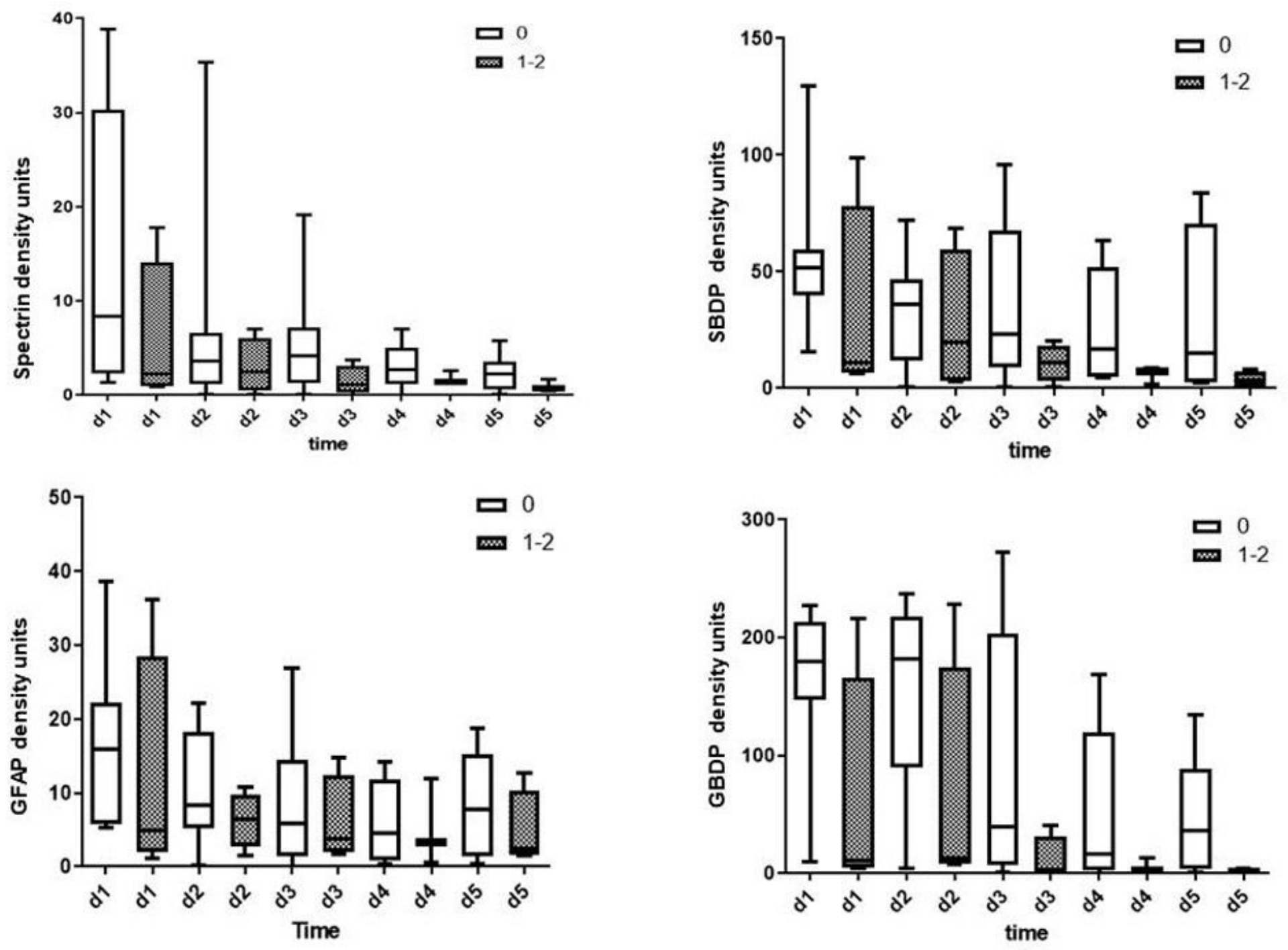

Figure 3

Ability of CSF biomarker levels in predicting AIS improvement (discharge AIS - initial AIS) Biomarkers measured with Immunoblotting. Quantification shown as mean + SEM for alpha II-spectrin, SBDP150/145, GFAP and GFAP-44K-38K. *, $* * * * \star p$ values $<0.05,0.01,0.001$, respectively between groups. 

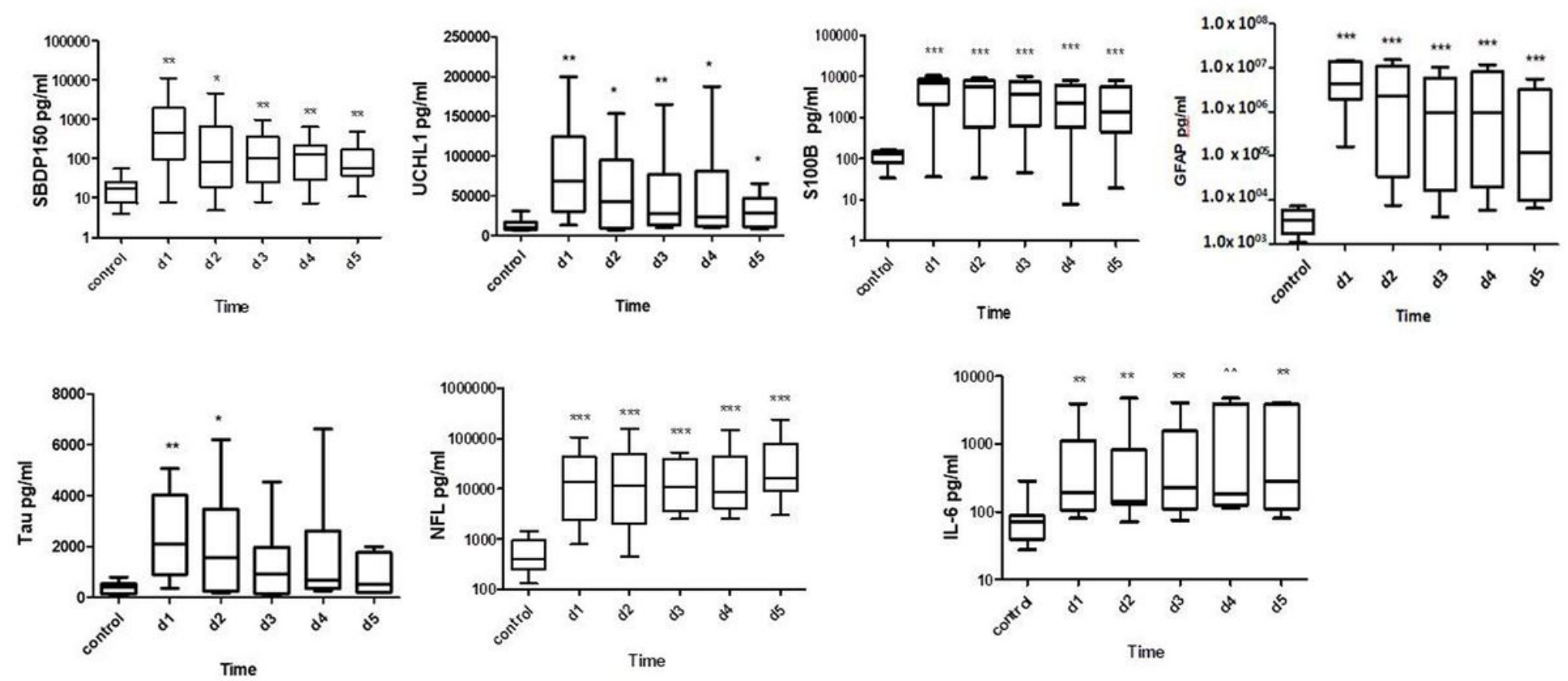

\section{Figure 4}

The expression of CSF biomarkers in acute injury phase in SCI patients by ELISA Quantification shown as

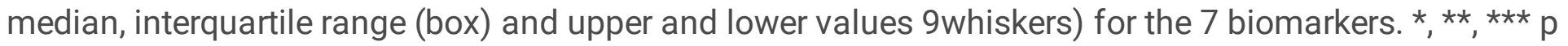
values $<0.05,0.01,0.001$, respectively vs. control.
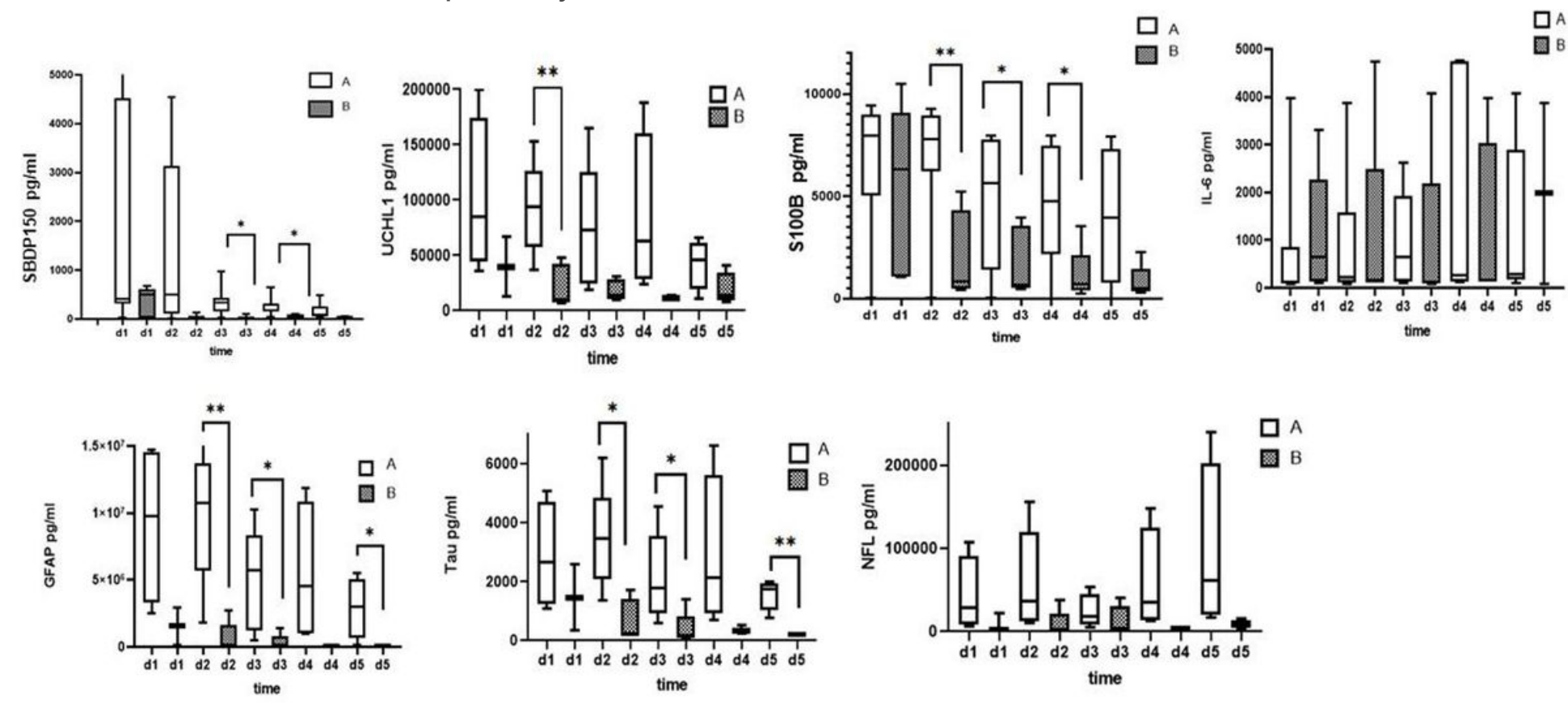

Figure 5

Correlation of CSF biomarker values to injury severity (initial AIS) Biomarkers measured by ELISA. Quantification shown as median, interquartile range (box) and upper and lower values 9 whiskers) for the

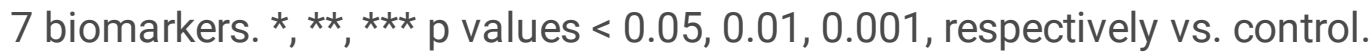



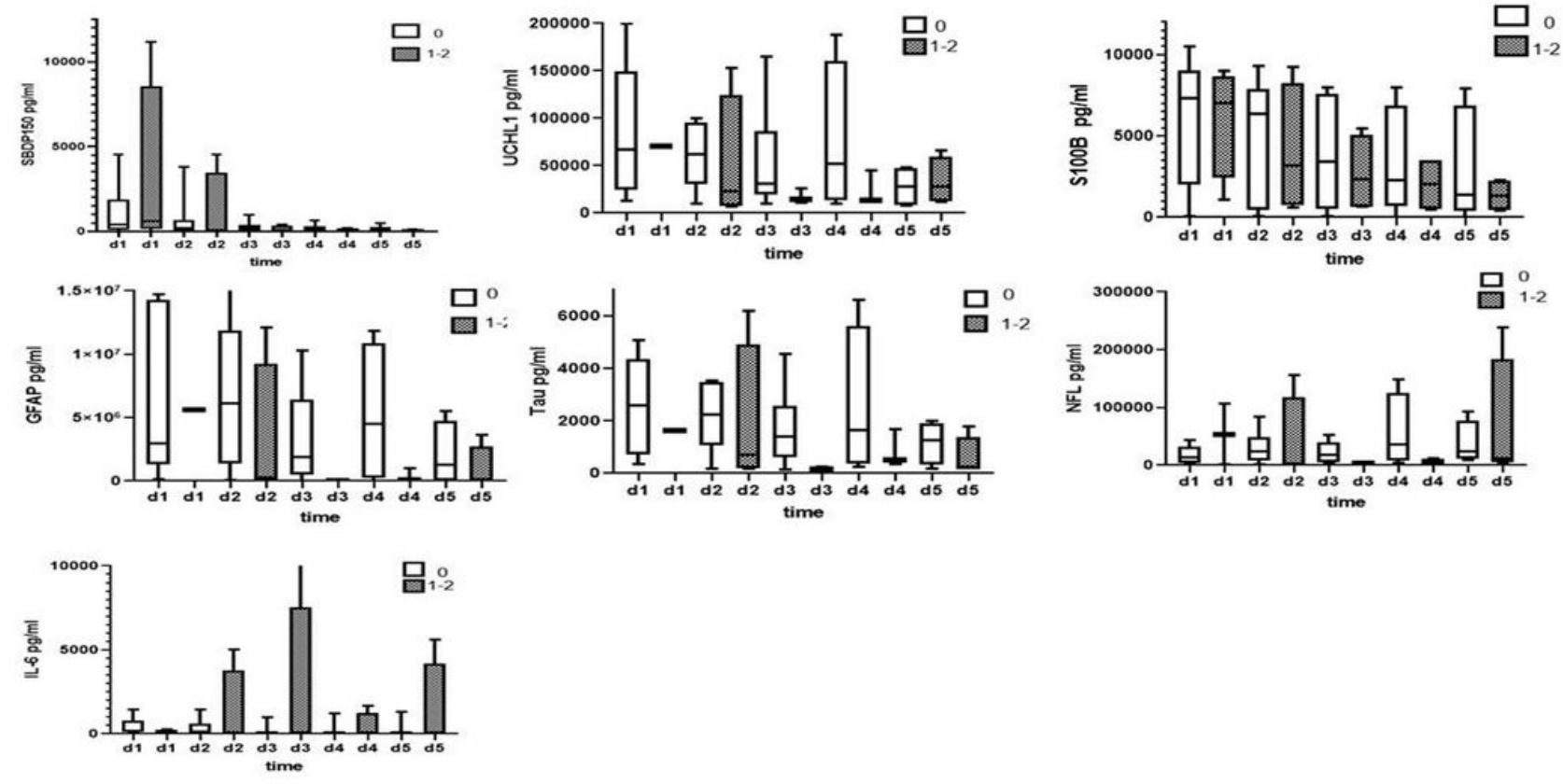

Figure 6

Ability of CSF biomarker values in predicting AIS improvement (discharge AIS - initial AIS) Biomarkers measured with ELISA. Quantification shown as median, interquartile range (box) and upper and lower values 9 whiskers) for the 7 biomarkers. ${ }^{*}, \star \star, * \star *$ p values $<0.05,0.01,0.001$, respectively vs. control. 

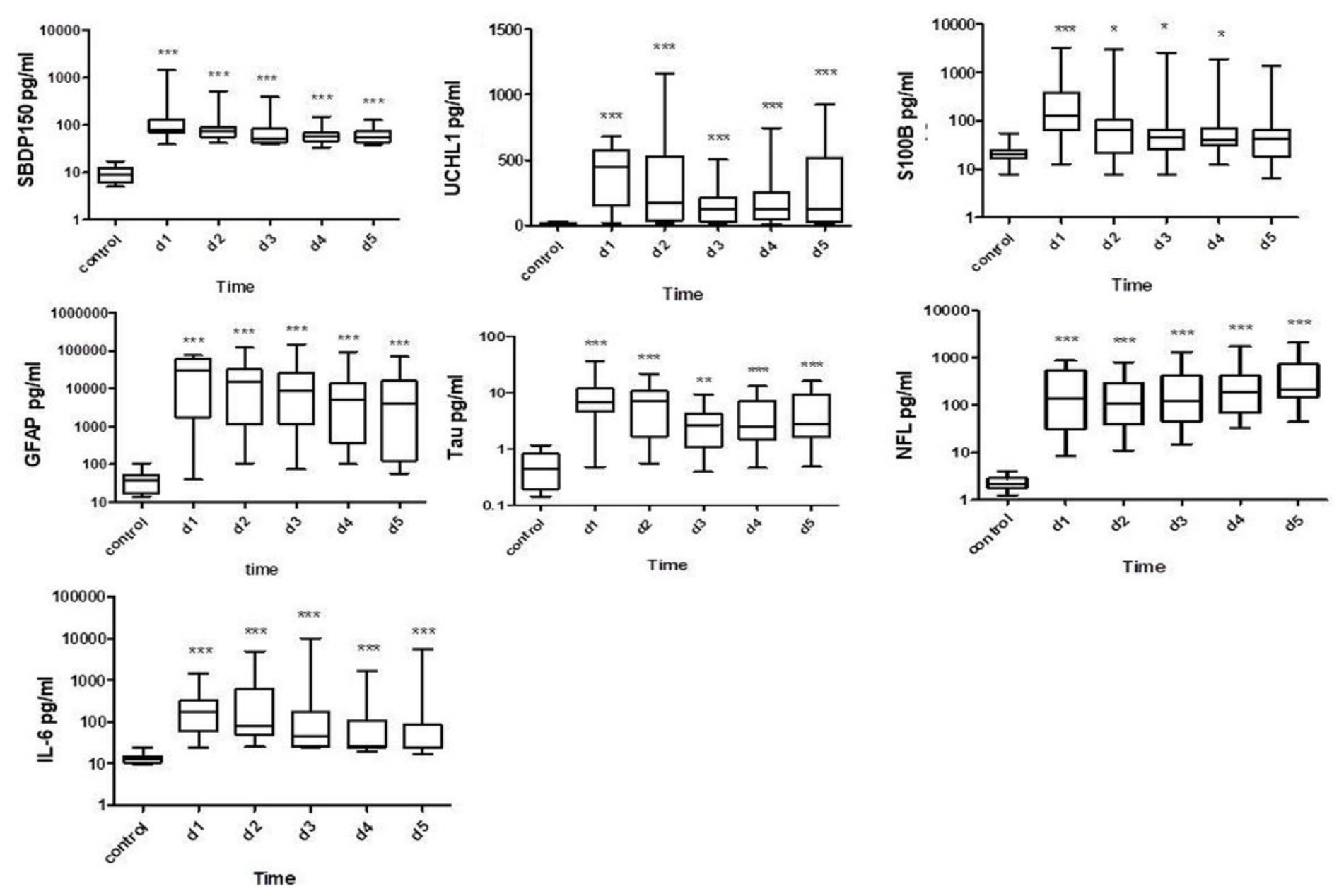

Figure 7

The expression of serum biomarkers in acute injury phase in SCI patients measured by ELISA Quantification shown as median, interquartile range (box) and upper and lower values 9 whiskers) for the

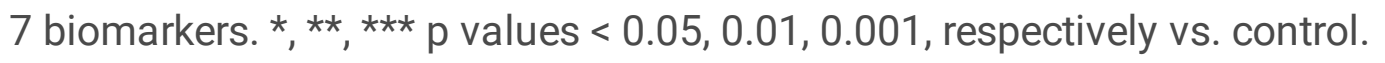




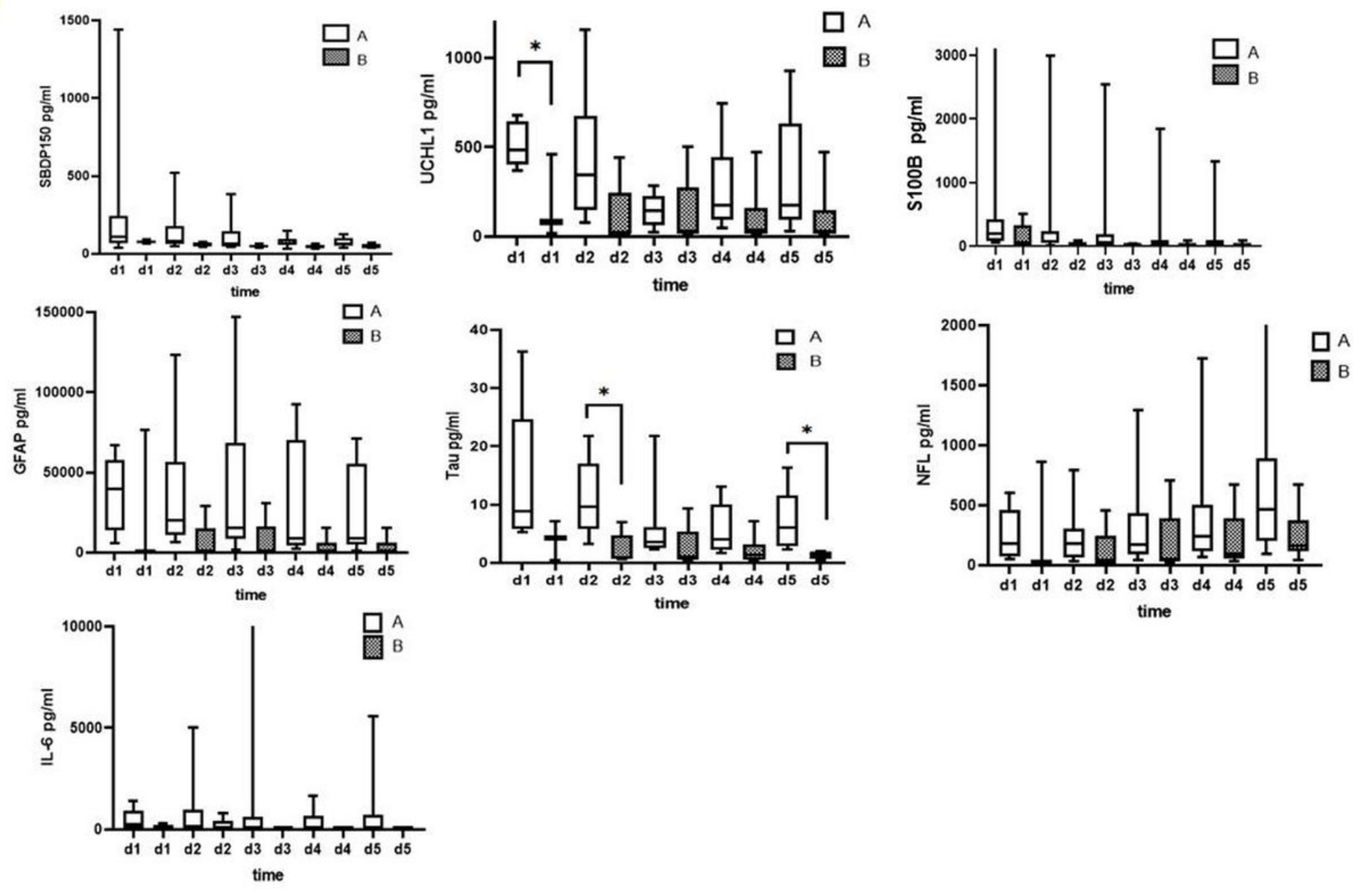

Figure 8

Correlation of serum biomarker values to injury severity (initial AIS) Biomarkers measured by ELISA. Quantification shown as median, interquartile range (box) and upper and lower values 9 whiskers) for the 7 biomarkers. ${ }^{*}{ }^{\star \star},{ }^{\star \star \star} \mathrm{p}$ values $<0.05,0.01,0.001$, respectively vs. control. 

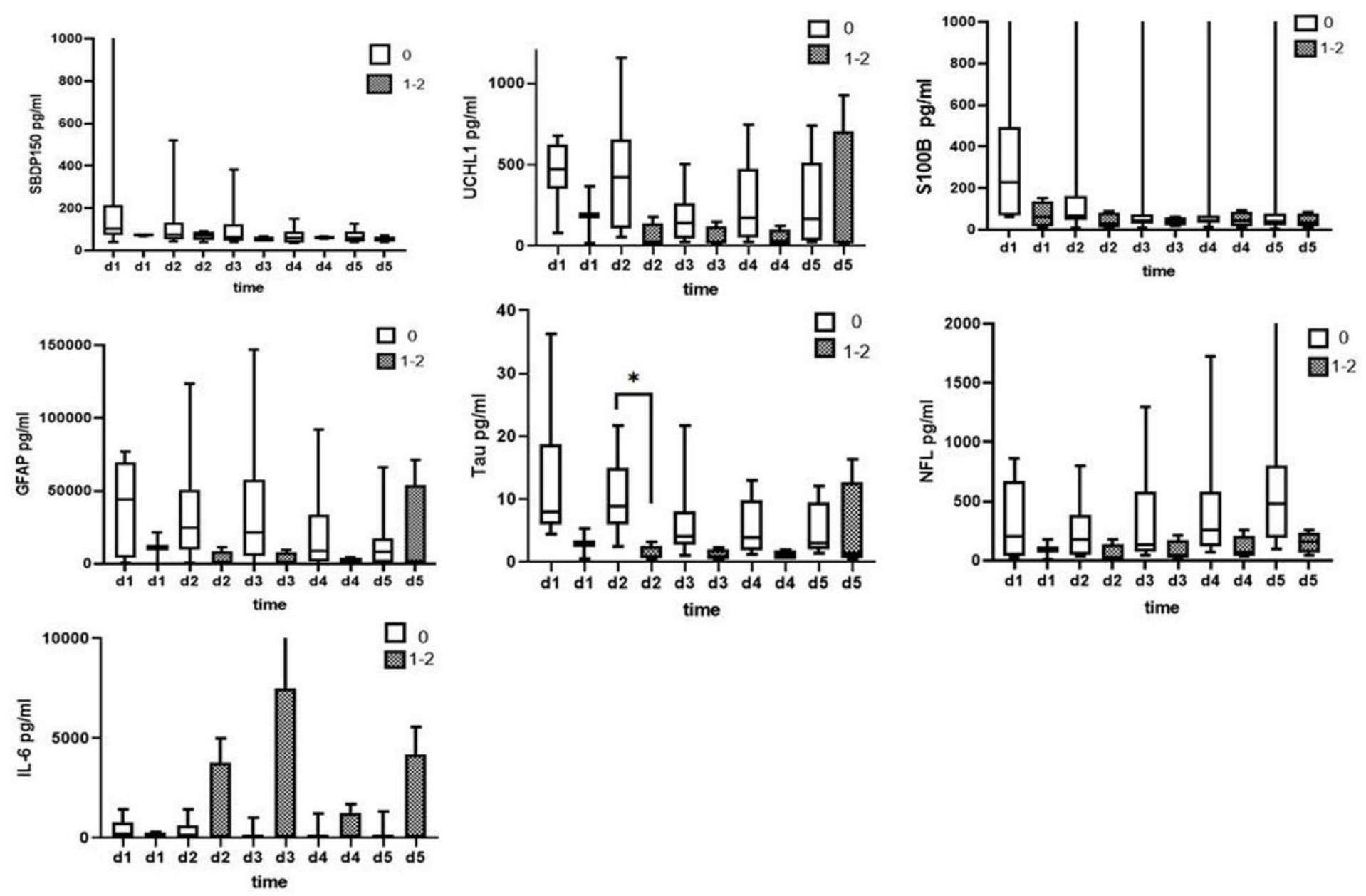

Figure 9

Ability of serum biomarker values in predicting AIS improvement (discharge AIS - initial AIS) Biomarkers measured with ELISA. Quantification shown as median, interquartile range (box) and upper and lower values 9 whiskers) for the 7 biomarkers. ${ }^{*}, * \star, * \star *$ p values $<0.05,0.01,0.001$, respectively vs. control.

\section{Supplementary Files}

This is a list of supplementary files associated with this preprint. Click to download.

- OnlineResource1.tif

- OnlineResource2.docx

- OnlineResource3.tif

- OnlineResource4.tif

- OnlineResource5.tif

- OnlineResource6.docx

- OnlineResource7.tif 